\title{
Mapping Techniques for Aligning Sulci across Multiple Brains
}

\author{
Duygu Tosun ${ }^{1}$, Maryam E. Rettmann ${ }^{2}$, and Jerry L. Prince ${ }^{1}$ \\ 1 Department of Electrical and Computer Engineering \\ Johns Hopkins University, Baltimore, MD, 21218, USA \\ 2 Gerontology Research Center, National Institute on Aging \\ National Institutes of Health, Baltimore, MD, 21224, USA \\ \{duygu, rettmann, prince\}@iron.ece.jhu.edu \\ http://iacl.ece.jhu.edu/index.html
}

\begin{abstract}
Visualization and mapping of function on the cortical surface is difficult because of its sulcal and gyral convolutions. Methods to unfold and flatten the cortical surface for visualization and measurement have been described in the literature. This makes visualization and measurement possible, but comparison across multiple subjects is still difficult because of the lack of a standard mapping technique. In this paper, we describe an approach that maps each hemisphere of the cortex to a portion of a sphere in a standard way. To quantify how accurately the geometric features - i.e., sulci and gyri - are mapped into the same location, sulcal alignment across multiple brains are analyzed, and probabilistic maps for different sulcal regions are generated to be used in automatic labelling of segmented sulcal regions.
\end{abstract}

\section{Introduction}

Understanding the relationship between the structure and function of the human brain cortex is a primary goal in human brain mapping. Visualization and localization of function on the cortical surface, however, is difficult because of the extensive sulcal and gyral convolutions and their variability between individuals. Cortical unfolding procedures expose the buried folds of cortical gray matter, to reveal the entire structure of part or all of the cortex on a flat, convex, or radial surface 1/2]34/5]6]. Preservation of the metric details - i.e., creation of approximately isometric maps - of the 3D surface has been a major goal in flattening [5:12]. With these maps, visual assessment and measurements of geometric relationships - e.g., distances, angles, areas — are similar to the original surface. Because it is impossible to generate an exact isometric map from the convoluted cortical surface, several mapping approaches use "cuts" in the surface, which are generally made manually [5. This makes it difficult to establish a "standard" coordinate system for cortical mapping because the shape of the final map is dependent on the cut that is made.

A more recent goal in cortical unfolding is to create maps that are in a standardized coordinate system, in the sense that they put known anatomical 
features - e.g., sulci and gyri — at the same coordinates in the mapped space 7 , 89]. Some methods enforce manually identified corresponding features to correspond on the computed maps [8]9]. Others derive correspondence by maintaining strict point correspondences between parametric models that are initialized and deformed in similar fashion throughout the surface estimation process [7].

Our long-term goal is to derive a standardized cortical map that does not require strict point correspondences throughout the estimation process and does not require manually identified landmarks. In this paper, we present a first step in this direction. Our approach combines parametric relaxation, iterated closest point registration, and conformal mapping. While no single one of these steps is new by itself, the specific implementation and combination of the methods is unique, and we also provide an extensive validation of the performance of the algorithm in providing automatic labelling of specific sulci - central, cingulate, superior frontal, and parieto-occipital.

\section{Background}

Brain Surface. Starting from T1-weighted SPGR [10] volumetric axially acquired magnetic resonance (MR) images acquired by the Baltimore Longitudinal Study of Aging [11] the surface that lies halfway between gray matter-white matter and gray matter-cerebrospinal fluid interface is reconstructed using a previously described method [12], along with several improvements [13]. Each final reconstruction consists of a triangular mesh comprised of approximately 300,000 vertices.

Conformal Mapping. Angenent et al. 4] proposed a way to construct a conformal flattening equivalence as the solution of a second-order elliptic partial differential equation on the 3D surface to be flattened. Solution of this partial differential equation represents an analytic procedure to map a cortical surface to the complex plane; for triangulated surfaces, a numerical procedure involving a finite element approximation can be used. A map to the unit sphere is achieved using the inverse stereo-graphic projection while keeping the overall map conformal.

This technique unfolds the convoluted brain surface while preserving relative angles, local shape, and connectivity of the triangulated surface. Other conformal maps can be generated, however, either by setting up the conformal coordinates on the cortical surface differently, or by applying coordinate reflections and Mobius transformations on the points mapped to the complex plane. We have used both coordinate reflection to correctly orient left/right and front/back and Mobius transformation to reduce area distortion on the resulting map [6]. Our maps yield either maps of the entire cortical surface to the sphere or maps of each cortical hemisphere to a portion of a sphere with a cone removed. 


\section{Methods}

We start from a triangle mesh representation of the cortical surface. The simplest (and oldest) way to establish spherical coordinates on one of these surfaces is to iteratively deform the vertices of the triangle mesh until they can be mapped to a sphere [5]14]. This basic approach is at the heart of many pseudo-isometric mapping methods. We start with this approach as well, but stop at the stage of a partially flattened map and then go directly to a spherical or hemispherical map using conformal mapping. There is no expectation, of course, that the total map will be conformal, but that is not our aim.

It is possible to go directly from the initial cortical geometry to the sphere using conformal mapping, but we have found that the precise details of the resulting map are very sensitive to the triangulation of the surface itself and to the conformal coordinates (primarily the choice of the "north pole") selected on the triangulated surface. We have found that initial iterative relaxation produces a more uniform triangle mesh, and at the stage of a partially flattened map, it yields a more consistent conformal map to the sphere. This is the first method we describe; the second method is a variation on this theme. At the stage of a partially-flattened map — arrived at by a shape-based stopping criterion evaluated during iterative relaxation - it is straightforward to register the subject surface to an atlas surface and project the subject vertices to the atlas surface. Each projected vertex is then transformed to the sphere (or hemisphere) using a single conformal map derived from the atlas surface. It turns out that this latter map yields a very consistent standardized coordinate system, not requiring manually-derived cortical features. We now describe each of these steps leading to the two described methods.

Surface Inflation. To obtain a more regular mesh structure, the vertices are iteratively repositioned according to

$$
V_{i}^{t+1}=(1-\lambda) V_{i}^{t}+\lambda \bar{V}_{i}
$$

where $V_{i}$ is the position of the $i^{\text {th }}$ vertex, $\bar{V}_{i}$ is an "average" vertex position, $t$ is the iteration number, and $\lambda \in[0,1]$ is a smoothing parameter. Let $N_{i}$ be the set of all triangles that contains vertex $V_{i}$, and let $C_{j}$ be the center of the triangle $j$, and $A_{j}$ be its area. Then

$$
\bar{V}_{i}=\frac{1}{\sum_{j \in N_{i}} A_{j}} \sum_{j \in N_{i}} A_{j} C_{j}^{t} .
$$

Our goal is to stop relaxation at the point of a "partially flattened" surface that should be comparable across individuals. Our stopping criterion is based on the $L_{2}$ norm of mean curvature, $\|H\|_{2}$ [15], which is defined as

$$
\|H\|_{2}=\frac{1}{4 \pi} \sqrt{\int H^{2} d A},
$$

where $\mathrm{H}$ is the mean curvature. The measure $\|H\|_{2}$ is approximated by discrete differential geometry operators described in [16]. We stop relaxation when $\|H\|_{2} \leq \beta$ for a pre-selected $\beta$. 
The top row of Fig. 11 shows an initial cortical surface and several stages during relaxation corresponding to stopping criteria of $\beta=0.1, \beta=0.075$, and $\beta=0.05$. The corresponding distributions of triangle angles in the meshes are shown below the figures. A regular mesh should have angles more nearly equal to 60-degrees, which is apparently well-satisfied by all three inflated maps. In order to preserve some anatomical detail representing the presence of major sulci, we have selected $\beta=0.1$ as a stopping criterion in this paper. In future work, we expect to optimize this selection rather than using this arbitrary choice.

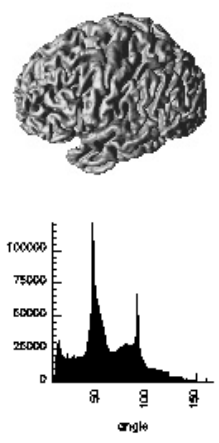

(a)
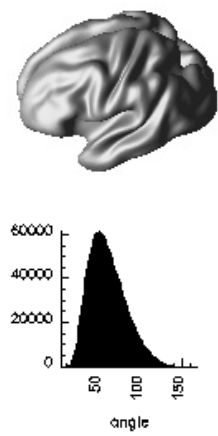

(b)
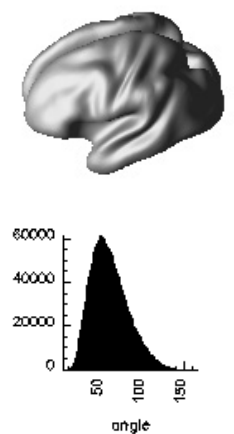

(c)
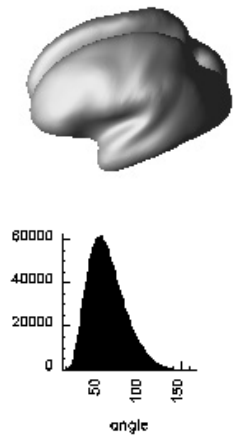

(d)

Fig. 1. Surface inflation: (a) Original cortical surface, (b) $\|H\|_{2} \leq 0.1$, (c) $\|H\|_{2} \leq$ $0.075,(\mathrm{~d})\|H\|_{2} \leq 0.05$

Surface Registration. As described previously, conformal mapping can be applied at any stage to map a surface to the sphere or hemisphere 6]. Since triangle regularity of the meshes is quite good after iterative relaxation, the conformal mapping is less sensitive to its initial conditions. But it is still necessary to pick a "north pole" for the conformal mapping of a surface so that it corresponds to a common position across all brains - these north poles will map to the same point on the sphere. To choose a good common north pole, we have selected one brain out of our database to be an "atlas brain." All other inflated surfaces are registered to the inflated surface of our atlas brain using a modified iterated closest point (ICP) algorithm. The ICP modification is that the corresponding closest point to a given surface vertex is computed as its projection onto the other surface, and this is done symmetrically for all vertices of both surfaces. The north pole of the atlas brain, selected at the top of the corpus callosum crossing the hemispheres is then projected to the subject brain and used as the subject north pole for conformal mapping.

Spherical Mapping. The preceding alignment procedure yields a common north pole so that a given inflated surface can generate its own conformal map to the sphere or hemisphere. The method described in [6] utilizes reflections and Mobius transformations to guarantee that the orientation of the mapped surface is correct and that its area distortion relative to the original surface is minimized 
(within the group of Mobius transformations). We found in our evaluations, however, that this approach — that of producing a separate conformal map for each subject — does not yield good alignment on either the spherical or hemispherical maps (see Section 4).

A second approach is readily developed using the single optimized conformal map of the atlas brain inflated surface. Since each subject's inflated surface is registered to the atlas, and all surfaces have very similar overall shape due to the mean curvature stopping criterion, it is possible to map all subject inflated surface vertices to the atlas inflated surface. Then, since the atlas's conformal map is a continuous bijection defined on the entire surface of its triangle mesh, all these points are readily mapped to the sphere (or hemisphere) using the atlas map, which is a pseudo-conformal map. In this way, to the extent that regions - e.g., sulci and gyri — are well-aligned on the inflated surfaces, they are guaranteed to be well-aligned on the spherical maps. This approach proves to be quite reliable, as shown in the following section.

\section{Results and Discussion}

In this section, we evaluate how common brain features map to similar locations on the sphere under the described mapping techniques. We start by analyzing the locations to which four sulci are mapped in 30 brains. We then demonstrate the power of a simple sulcal labelling technique on 5 brains given the probabilistic map created from the 30 training brains.

Probabilistic Maps. We randomly picked 30 brain surfaces from our database of 35 surfaces. Four sulcal regions, defined as the buried cortex surrounding the sulcal spaces, were automatically segmented [17] and manually labelled on all 35 brains. These sulcal regions are the central sulcus (cs), sylvian fissure (sf), cingulate sulcus (cn) and parieto-occipital sulcus (po) on both the left and right cortical hemispheres.

Let $\mathrm{HS}_{r}$ denote the reference hemisphere to which all brains are mapped. (This hemisphere is parameterized by the particular conformal map of the atlas brain, which is just one of the 30 brains picked randomly.) To see where the four sulci map to on $\mathrm{HS}_{r}$, we generated four probabilistic maps $I_{i}: H S_{r} \rightarrow[0,1]$ for $i=\mathrm{cs}$, sf, cn, and po. For each sulcal region, $I_{i}$ is initialized to zero, and for each vertex of $H S_{r}$, the value of probabilistic map is incremented by 1 for each training brain, if the corresponding point on the hemispherical map of that brain has the same sulcal label. Then $I_{i}$ is divided by 30 to normalize its values to the range $[0,1]$. This gives a probabilistic map comprising an estimated conditional probability of the location of a sulcal region for each of four sulcal regions.

Two probabilistic maps were generated, one for the map $I^{(1)}$ created using individual conformal maps, and one for the map $I^{(2)}$ created by using only the atlas conformal map. Fig. 2] shows the probabilistic maps $I_{i}^{(2)}$ for $i=\mathrm{cs}$, sf, cn, po for both left and right cortical hemisphere. The maximum value of probabilistic maps $I_{i}^{(1)}$ and $I_{i}^{(2)}$ are given in Table (1). The table reveals a lack of good sulcal alignment for the multiple conformal maps approach.

Automatic Sulcal Labelling. Here, we examine the performance of a simple automated sulcal labelling technique using the probabilistic maps derived in the 
Table 1. Maximum of Probabilistic Maps

\begin{tabular}{|c|c|c|c|c|c|c|c|c|}
\hline & \multicolumn{6}{|c|}{ Left cortical hemisphere } & \multicolumn{4}{|c|}{ Right cortical hemisphere } \\
\hline Mapping & cs & sf & cn & po & cs & sf & cn & po \\
\hline$I^{(1)}$ & 0.72 & 0.74 & 0.88 & 0.72 & 0.61 & 0.61 & 0.94 & 0.78 \\
\hline$I^{(2)}$ & 0.97 & 0.90 & 0.97 & 1.00 & 0.97 & 0.90 & 1.00 & 0.97 \\
\hline
\end{tabular}

previous section. Let $I_{i}$ be one of the probabilistic maps derived in the previous section using the 30 training set surfaces. Now consider one the 5 remaining test surfaces, each of which is mapped to a hemisphere using either the first or second approach. For each segmented sulcal region on the test surface, the maximum of each probabilistic map is calculated. If the maximum is larger than 0.5 , then the label of that probabilistic map is assigned to the entire sulcal region.

Fig. 33a shows a mapped sulcal segmentation. The correct manual labels for the four sulcal regions under consideration are given (for the left hemisphere) in Fig. 3b. Fig. 3r shows the result of the automated labelling technique.

To evaluate the effectiveness of this labelling technique, and hence the spherical mapping technique, two types of labelling errors are defined: probability of detection $P_{i}^{D}$ and probability of false alarm $P_{i}^{F}$. Probability of detection is defined as the percentage area of the manually labelled region that is correctly labelled by our automatic labelling method. Probability of false alarm is defined as the percentage area of the automatically labelled region that is not manually label. The probabilities of detection and false alarm averaged for the five test brains are reported in Table (2). Higher $P_{i}^{D}$ values and lower $P_{i}^{F}$ show that our second approach yields a better standardized coordinate system.

Table 2. Automatic labelling errors

\begin{tabular}{|c|c|c|c|c|c|c|c|c|}
\hline & \multicolumn{4}{|c|}{ Left cortical hemisphere } & \multicolumn{4}{|c|}{ Right cortical hemisphere } \\
\hline Errors & cs & sf & cn & po & cs & sf & cn & po \\
\hline$P_{i}^{D}$ by $I_{i}^{(1)}$ & 0.93 & 0.81 & 0.83 & 0.90 & 1.00 & 0.94 & 0.89 & 0.92 \\
\hline$P_{i}^{F}$ by $I_{i}^{(1)}$ & 0.34 & 0.23 & 0.04 & 0.17 & 0.32 & 0.22 & 0.08 & 0.37 \\
\hline \hline$P_{i}^{D}$ by $I_{i}^{(2)}$ & 1.00 & 0.86 & 0.91 & 0.98 & 1.00 & 1.00 & 1.00 & 1.00 \\
\hline$P_{i}^{F}$ by $I_{i}^{(2)}$ & 0.02 & 0.23 & 0.03 & 0.17 & 0.00 & 0.17 & 0.09 & 0.33 \\
\hline
\end{tabular}

\section{Conclusion}

We have developed a standard mapping technique that automatically produces mappings with good sulcal alignment across multiple brains. Our method depends only on the geometry of the surface. One of the primary goals of the proposed coordinate system is to reduce the spatial uncertainty associated with the 
(a)
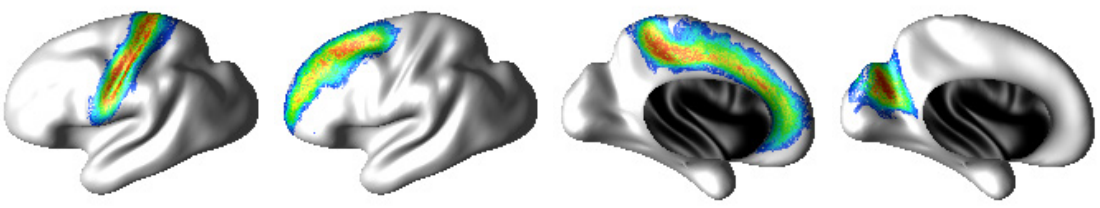

(b)
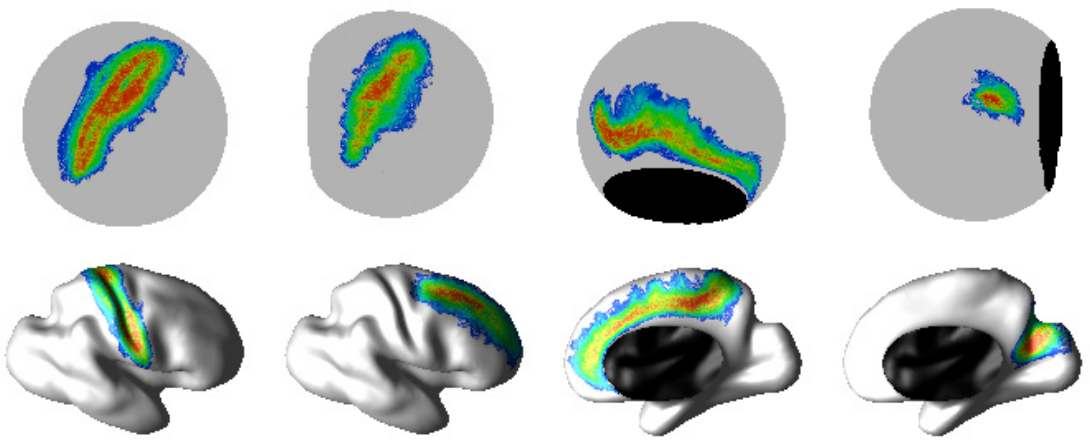

(c)

(d)

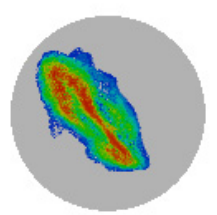

(cs)

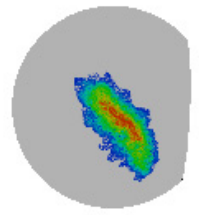

(sf)

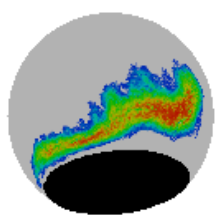

(cn)

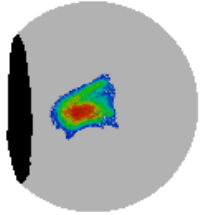

(po)

Fig. 2. Probabilistic maps $I_{i}^{(2)}$ on atlas inflated surface (a,c) and conformal map (b,d) for both left $(\mathrm{a}, \mathrm{b})$ and right $(\mathrm{c}, \mathrm{d})$ cortical hemispheres (color map: dark blue $(0)$ - dark $\operatorname{red}(1))$

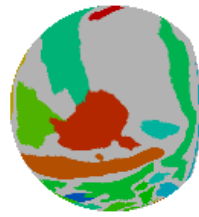

(a)

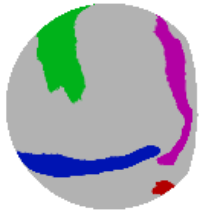

(b)

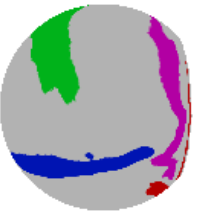

(c)

Fig. 3. Pseudo-conformal map of test brain (a) Sulcal segmentation (b) Manually labelled sulcal regions (c) Automatically labelled sulcal regions using $I^{(2)}$ (Color map for (b) and (c):Left cs (blue), left sf (green), left cn (pink) and left po (red))

location of a given anatomical or functional area. Incorporation of additional features should be considered to improve sulcal alignment on hemispherical maps, which can yield further improvements in automatic labelling of sulcal regions. 
Acknowledgments. We would like to thank Drs. Susan Resnick and Dzung Pham for access to and guidance in the use of the BLSA data. This work was supported by the NIH/NINDS under grant R01NS37747.

\section{References}

1. G.J.Carman, H.A.Drury, and D.C.Van Essen. Computational methods for contruction and unfolding the crebral cortex. Cerebral Cortex, 5:506-517, 1995.

2. H.A.Drury, D.C.Van Essen, C.H.Anderson, C.W.Lee, T.A.Coogan, and J.W.Lewis. Computerized mappings of the cerebral cortex: A multiresolution flattening method and a surface-based coordinate system. J.Cog.Neurosci., 8:1-28, 1996.

3. B.Wandell, S.Engel, and H.Hel-Or. Creating images of the flattened cortical sheet. Invest.Opth.and Vis.Sci., 36(612), 1996.

4. S.Angenent, S.Haker, A.Tannenbaum, and R.Kikinis. On the Laplace-Beltrami operator and brain surface flattening. IEEE Trans.Med.Imag., 18(8):700-711, 1999.

5. B.Fischl, M.I.Sereno, and A.M.Dale. Cortical surface-based analysis II: Inflation, flattening, and a surface-based cortical system. NeuroImage, 8(4):272-284, 1999.

6. D.Tosun, M.E.Rettmann, X.Tao, X.Han, C.Xu, and J.L.Prince. Calculation of human cerebral cortical thickness on opposing sulcal banks. In Proc. of rth Int. Conf. on Functional Mapping of the Human Brain (HBM), volume 4322, June 2001.

7. D.MacDonald, N.Kabani, D.Avis, and A.Evans. Automated 3D extraction of inner and outer surfaces of cerebral cortex from MRI. NeuroImage, 12(3):340-356, 2000.

8. P.M.Thompson, R.P.Woods, M.S.Mega, and A.W.Toga. Mathematical and computational challenges in creating deformable and probabilistic atlases of the human brain. Human Brain Mapping, 9:81-92, 2000.

9. X.Gu, Y.Wang, T.F.Chan, P.M.Thompson, and S.T.Yau. Genus zero surface conformal mapping and its application to brain surface mapping. submitted, 2003.

10. H.Z. Wang and S.J. Rieder. A spoiling sequence for suppression of residual transverse magnetization. Magn.Reson.Med., 15(2):175-91, 1990.

11. S.M.Resnick, A.F.Goldszal, C.Davatzikos, S.Golski, M.A.Kraut, E.J.Metter, R.N.Bryan, and A.B.Zonderman. One-year age changes in MRI brain volumes in older adults. Cerebral Cortex, 10(5):464-72, May 2000.

12. C.Xu, D.L.Pham, M.E.Rettmann, D.N.Yu, and J.L.Prince. Reconstruction of the human cerebral cortex from magnetic resonance images. IEEE Trans.Med.Imag., 18(6):467-480, 1999.

13. X.Han, C.Xu, and J.L.Prince. A topology preserving deformable model using level sets. In IEEE Workshop on Mathematical Methods in Biomedical Image Analysis (MMBIA2001), pages 213-220, Dec. 2001.

14. J.I.Sereno, A.M.Dale, A.Liu, and R.B.H.Tootell. A surface-based coordinate system for a canonical cortex. NeuroImage, 3:S252, 1996.

15. A.C.Smith, P.G.Batchelor, D.L.G.Hill, A.F.Dean, T.Cox, and D.J.Hawkes. The shape of the developing foetal cortex from MR images. Proc.Medical Image Understanding and Analysis(MIUA), 2000.

16. M.Meyer, M.Desbrun, P.Schroder, and A.H.Barr. Discrete differential geometry operators for triangulated 2-manifolds. VisMath'02 Proceedings, 2002.

17. M.E.Rettmann, X.Han, C.Xu, and J.L.Prince. Automated sulcal segmentation using watersheds on the cortical surface. NeuroImage, 15:329-344, February 2002. 